\title{
Literature Review of Cultural Competence Curriculum within the United States: An Ethical Implication in Academic Preparational Programs
}

\author{
Marla B. Hall, Jeffrey J. Guidry \\ Texas A\&M University, Department of Health \& Kinesiology, Division of Health Education
}

\section{ARTICLE INFO}

Received

: 26/05/2012

Accepted : :22/08/2012

Published : :01/03/2013

\section{KEYWORD}

Cultural competence

Academic preparational

programs

Curriculum development

\section{ABSTRACT}

Objective: The purpose of this literature review is to explore literature focused on health related preparational programs' ability, experiences and effectiveness in developing the skill sets of students to improve health outcomes of ethnic and racial minority populations. Method: EbscoHost, MEDLINE, PubMed and Google Scholar were searched for articles published between 1998 and 2012 to identify research on cultural competency academic curriculum in health disciplines. From this literature review, researchers extracted studies reported on cultural competency in collegiate programs and practitioner assessments. Result: Sixteen articles were identified, 10 original research studies and 6 review articles or commentaries. Of these, eight were from medicine, three from health education, two from nursing and three from pharmacy education. The majority of studies utilized a case study or multi-site assessment approach. Themes derived from the review include: administrative views and assessments; practitioner academic preparation analysis; student focused activities and; guided academic curriculum development. Conclusion: Groups currently experiencing poorer health status are expected to grow as a proportion of the total U.S. population; therefore, the future health of America as a whole will be influenced substantially by improving the health of these racial and ethnic minorities. As a result health education specialists must be culturally competent to implement effective programming targeting these populations. Cultural competence incorporates principles which contribute to understanding the cultural and social influence on individuals' health behaviors and beliefs. Those employed in preparation programs have a responsibility to build the capacity of students through the utilization of appropriate curriculum.

(c) Medical Education Department, School of Medical Sciences, Universiti Sains Malaysia. All rights reserved.

CORRESPONDING AUTHOR: Marla B. Hall, Texas A\&M University, Department of Health \& Kinesiology, Division of Health Education.

Email: mbh1120@hlkn.tamu.edu 


\section{Introduction}

The demographic changes anticipated over the next decade magnify the importance of addressing disparities in health status. Groups currently experiencing poorer health status are expected to grow as a proportion of the total U.S. population; therefore, the future health of America as a whole will be influenced substantially by improving the health of these racial and ethnic minorities [1]. In order to be effective with strategies, health education specialists must be culturally competent to implement beneficial programming targeting ethnic/racial minority groups. Cultural competence is defined as awareness of unique characteristics of ethnic/racial minority populations serviced by health professionals in clinical and non-clinical settings [2]. Cultural competence incorporates various principles that contribute to understanding the cultural and social influence on individuals' health behaviors and beliefs; and the transcendent journey into health education practice.

According to the Coalition of the National Health Education Organizations the Code of Ethics for the Health Education Practice's Article VI suggests educators have a responsibility in conveying effective strategies to students within preparational programs [3]. Specifically it states, "Those involved in the preparation and training of health educators have an obligation to accord learners the same respect and treatment given other groups by providing quality education that benefits the profession and the public [3]." It can be argued the concept of cultural competence fits within this code because preparing future practitioners will ensure the inclusion of best practices for minority health program and/or intervention methods, benefiting the population. This fosters ethnic/minority racial group participants' likelihood of adopting and sustaining healthy behaviors being addressed [3]. Subsequently, it will be an asset to the profession by enabling valid research methodology and duplication. Due to research being scarce in the field of health education, this paper seeks to explore literature relating to multidisciplinary preparational programs' ability, experiences and effectiveness in developing the skill sets of students to engage in improving health outcomes of racial and ethnic minority groups.

\section{Method}

EbscoHost, MEDLINE, PubMed and Google Scholar were searched for articles published between 1998 and 2012 to identify research on cultural competency academic curriculum in health disciplines. From this literature review, researchers extracted studies reported on cultural competency in collegiate programs and practitioner assessments. Sixteen articles were identified, 10 original research studies and 6 review articles or commentaries. Of these, eight were from medicine, three from health education, two from nursing and three from pharmacy education. The majority of studies utilized a case study or multi-site assessment approach. Themes derived from the review include: administrative views and assessments; guided academic curriculum development; student focused activities and; research training and curriculum development. The findings of the case studies and surveys are further discussed with a review of the literature. The matrix illustrated in Table 1 provides a synopsis of the core themes represented in each paper. 
Table 1. A synopsis of core themes found in each paper

\section{AUTHORS AND YEAR OF PUBLICATIONS}

\begin{tabular}{|c|c|c|c|c|c|c|c|c|c|c|c|c|c|c|c|c|}
\hline $\begin{array}{l}\text { THEMES } \\
\text { COVERED IN } \\
\text { EACH } \\
\text { PAPER }\end{array}$ & 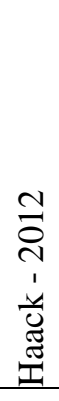 & 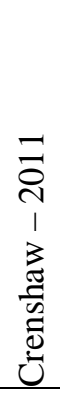 & 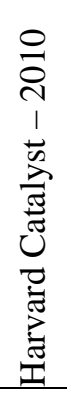 & 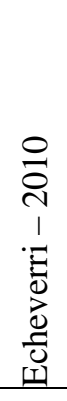 & 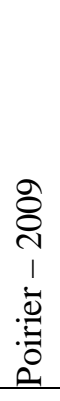 & 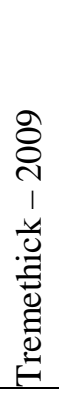 & 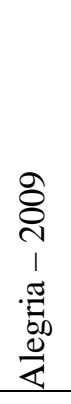 & 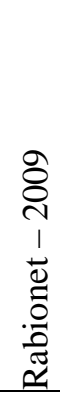 & 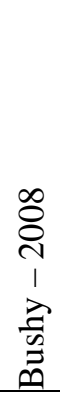 & 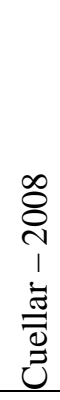 & 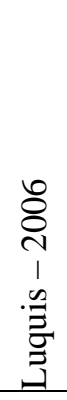 & 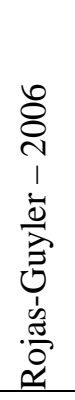 & 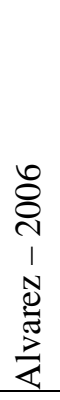 & 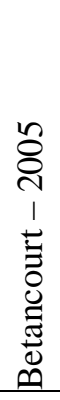 & 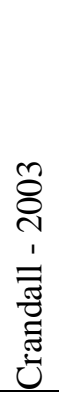 & $\begin{array}{l}\text { क् } \\
\frac{1}{7} \\
\frac{1}{0}\end{array}$ \\
\hline $\begin{array}{l}\text { Administrative } \\
\text { Views and } \\
\text { Assessment }\end{array}$ & & & • & & & & & & & & • & & & & & \\
\hline $\begin{array}{l}\text { Practitioner } \\
\text { Academic } \\
\text { Preparation } \\
\text { Analysis }\end{array}$ & & & - & & & & & & & & & • & & & & \\
\hline $\begin{array}{l}\text { Student Skills } \\
\text { Building } \\
\text { Activities }\end{array}$ & - & & & & • & • & & & & • & & & & & • & \\
\hline $\begin{array}{l}\text { Research } \\
\text { Training }\end{array}$ & & & • & & & & • & $\bullet$ & • & & & & • & & & \\
\hline $\begin{array}{l}\text { Guided } \\
\text { Academic } \\
\text { Curriculum } \\
\text { Development }\end{array}$ & & - & • & $\bullet$ & • & & & & & • & & & & • & & - \\
\hline
\end{tabular}

\section{Result}

\section{Educators and Administrators}

Luquis, Perez and Young utilized surveys to assess current opportunities in health education preparational programs in the area of cultural competence, with a final recruitment of 157 participants. Academic institutions, offering both undergraduate and graduate degree programs in health education were selected, by utilization of the American Association of Health Education
(AAHE) Directory of Institutions [4]. The researchers obtained information on the number of courses in each program focusing on cultural competency, the percentage of capstone or core courses that integrate cultural competence, as well as the skills and content addressed in the courses. In addition, the assessment included questions regarding the level of involvement of faculty members with ethnic/racial minority groups; and their, as well as students', level of knowledge, comfort and commitment in the area [4]. 
According to the investigators, the findings indicated less than one-third of the programs offer a course devoted to cultural competency in its entirety. Eighty percent reported they do not provide cultural/diversity training or education programs for their faculty members. Many participants noted their course prepare students to design health education programs for diverse populations. Approximately $70 \%$ of the chairs or program coordinators reported faculty members in their department were committed to cultural competency and had high levels of comfortability in addressing the topic [4]. It was concluded from the analysis of the survey, there is a need for cultural competency required courses for students, cultural competence training and continuing education for faculty and staff and; specific standards for cultural competency among health education preparational programs [4].

\section{Practitioners}

Rojas-Guyler, Wagner and Chockalingam explored the Latino cultural competence among 110 health education specialists to evaluate the professional preparation implications. Specifically, the author assessed the level of cultural competence, attitudes, knowledge, barriers and acquisition process, to investigate recommendations for future professional development, through a survey instrument [5]. Roughly $90 \%$ of participants were both female and Caucasian, with the entire survey pool comprised of $38 \%$ undergraduate degree recipients, $40 \%$ having masters degrees and $11 \%$ with doctorates. The authors reported the majority of participants knew the significance cultural competence has in the profession. However, they face several barriers when attempting to plan, implement and evaluate programs for the Latino community. Some of the issues include the lack of knowledge by professionals in their organizations, limited access or contact with community members; and limited resources and educational opportunities [5].

Rojas-Guyler et al. also noted as it relates to their comfort level in working with Latino populations, between $39 \%$ and $44 \%$ of respondents identified as being "neutral" and over $22 \%$ noted being "uncomfortable". As expected, professionals who felt more comfortable with the implementation of health education programs in Latino communities had high levels of knowledge about the population [5]. Nearly $90 \%$ of those surveyed stated they did not receive enough knowledge about the Latino culture while pursuing degrees in health education. Most respondents expressed Latino cultural competence should be required as part of health education preparation at both the undergraduate and graduate levels. However, it was noted it should not be a single focus area, rather incorporated into a comprehensive, skill development curriculum [5].

\section{Student Skill Development}

Tremethick and Smit implemented a five year Honduran cultural immersion service-learning program to evaluate its effectiveness in facilitating cultural competency within a Midwest university, Health Education student population [6]. The objectives of the program were to allow participants the opportunity to conduct primary and secondary assessments, develop culturally appropriate programming based on assessments and; implementation and evaluation of programming in Honduras. The researchers implemented several service learning activities for students, beginning with a needs assessment in a local town. This entailed the observation of the population and interviews with community leaders [6]. All activities took place in an elementary school setting, which enrolled some of the poorest children in the area. Health Education students were required to convey lessons on basic hygiene, disseminating bars of soap, toothbrushes and toothpaste. There were also learning modules related to head lice prevention and treatment, with similar programming provided to single mothers residing in a nearby shelter [6].

In addition, students interacted with Hondurans in a local clinic waiting room, in an effort to observe babies and children's level of development and interactions between family 
members. The clinic was also used to implement folic acid programming to women waiting for clinical services. Advocacy was a major part of the service-learning experience as well. This allowed students to attend governmental meetings and media outlets for the development of a medical center, to increase health care access for residents of a poor, rural area of Honduras [6]. Tremethick and Smit stated at the end of the third year of the program, 36 students completed a qualitative and quantitative questionnaire to evaluate the course. Students reported they valued information from other cultures in understanding their own health and learned an abundance of information about the Honduran culture [6]. Data analysis also suggested participants felt they are more sensitive to cultural diversity and have the ability to apply the knowledge gained during their professional career. In addition, by implementing health education interventions in a developing country, students determined they acquired a better grasp of addressing barriers through program planning [6].

Poirier et al. designed, implemented and evaluated a pilot cultural competence course for pharmacy students. The objectives were to develop skills, increase awareness of personal biases and establish a cross-cultural appreciation of health belief differences. Researchers addressed these objectives by using a team-based learning instructional technique. These incorporated group presentations, panel discussions, portfolio reflections and readiness assessment tests [7]. Comparative analysis of pre- and post- outcomes using the Inventory for Assessing the Process of Cultural Competence among Healthcare Professionals (IAPCC-R) suggested an enhanced progress toward cultural competence acquisition [7]. Students reported a heightened level of awareness of personal biases, increased cultural proficiency and knowledge of health belief models and its effective application to patient care [7].

Crandall, George, Marion and Davis used a similar pilot approach by developing a communication and development theories-based year long cultural competency training course for second year medical students. Beyond traditional lectures, faculty employed the inclusion of guest speakers with expertise in the subject matter; critical and reflective journal entries; and service learning projects [8]. Evaluation suggested the course successfully improved skills, knowledge and attitudes as it relates to culturally competent care. It also resulted in a positive modification of the medical school's curriculum development and implementation approach [8].

Haack and Phillips investigated the incorporation of cultural competency in a Pharmacy Skills and Application course series. The researchers further assessed the level of competency among students regardless of participation. The activities of the course included self-reflection, case studies, diversity service learning, discussion and traditional lecture [9]. IAPCC-R was also used in this study to measure the cultural competence of both student populations. The findings suggested students with course series completion displayed higher scores in the cultural skills and cultural encounters subscales [9].

\section{Discussion}

\section{Research Training}

Alegria suggests training new researchers should involve strategies that are atypical from traditional curriculum, which include community-based participatory research (CBPR) and cultural competency [10]. The author notes this method will allow students or trainees the opportunity to explore innovative research and data collection methods to be modified to the needs of diverse populations. Alvarez et al. suggests researchers utilize a community organization outreach approach when attempting to increase minority participation. Many of the strategies used by the researchers in the study, which produced positive results, are comparable to CBPR and cultural competency techniques [11]. These include implementing programming in the community which is deemed necessary by the participants, and partnering with community leaders and/or bilingual and bicultural staff to support recruitment and retention efforts [10]. In 
addition, the researchers noted by utilizing community organizations there was increased access to vulnerable and hard to reach populations [11]. These organizations are effective outlets to utilize in increasing minority participant recruitment and retention. It is of high importance for research teams to reach out to these agencies, to heighten external validity or generalizability, of research results [11].

Rabionet, Santiago and Zorilla noted most researchers do not receive adequate training in preparational programs to work with minority populations. The authors suggested that most enter the profession assuming the perspective of the majority [12]. This is further translated into health research instrumentation tools. Analysis suggests assessment tools are designed for Caucasians, and often times the research process is comprised because minority groups are being forced to fit their experiences to rigid instruments [13]. The use of these instruments then place minority groups in a position which is subjected to the majority's normative standards. As a result, new research is inspired, based on stereotypes culminated from inappropriate interpretations [13]. Researchers must be culturally competent, designing assessment tools which acknowledge ethnic/racial minority participants as cultural beings. This will provide data analysis with increased relevance to the populations of study [13].

\section{Guided Practicum Development}

Literature identified the lack of cultural competency of health professionals having a direct link to minority health disparities. Specifically, being proactive with addressing methodological challenges will contribute to the advancement of the profession, as it relates to best practices and improving minority health outcomes [14]. Betancourt et al. conducted interviews with experts in cultural competency in the academe, to assess their perspectives of the field in improving the quality of and eliminating minority health disparities in healthcare. Many of the informants expressed concern regarding stereotypic teaching strategies that did not emphasize the exploration of socioeconomic issues, research bias and empathy [15]. Researchers also suggested the need for a unified conceptual teaching framework to address the current variability and quality of preparational programs [15].

In order to guide curriculum development, Echeverri, Brookover and Kennedy assessed the perceived level of cultural competence of pharmacy students. Researchers evaluated the effectiveness of the Clinical Cultural Competency Questionnaire (CCCQ) to measure student's knowledge, skills, attitudes and clinical encounters among cross-cultural environmental settings [16]. The tool was deemed reliable and the data obtained was analyzed further to identify training needs to foster tailored academic programming [16].

Crenshaw et al. employed nominal group technique sessions in an effort to identify and organize cultural competence curriculum opponents to address cardiovascular disease disparities. The research team recruited community and academic physicians; residents and medical students for participant inclusion [17]. It was determined the curriculum should encompass four concept areas. These include: (1) patient's cultural background; (2) provider and health care, communication skills; (3) crossculture; and (4) resources to manage cultural diversity [17]. Crenshaw et al. noted their qualitative and quantitative approach allowed an evaluation of a consensus among stakeholders. Subsequently, this was deemed beneficial in guiding the processes of content development and prioritizing the information gained [17].

Due to the difficulty and time consumption resulting from creating a completely new curriculum, Cuellar, Brennan, Vito and de Leon Siantz explored the infusion of cultural competence components in an existing undergraduate nursing program. Researchers utilized the Blueprint for Integration of Cultural Competence in the Curriculum (BICCC) as a framework for implementation of teaching strategies and learning objectives to assist in clinical care proficiency among students [18]. BICCC focuses on defining and operationalizing 
the terms associated with cultural competence within educational standards of academic teaching; and practitioner skill sets building as determined by accrediting agencies [18].

\section{Conclusion}

There is a level of urgency to assist budding health professionals in learning specific skill sets, enabling them to work effectively with minority populations. Life expectancy and overall health have improved in recent years for most Americans, thanks in part to an increased focus on preventive medicine and dynamic new advances in medical technology [1]. However, despite notable progress in the overall health of the Nation, there are continuing disparities in the burden of illness and death experienced by ethnic/racial groups. For many in these populations, good health is elusive, because of inappropriate health programmatic methods [14].

The American Association for Health Educators noted in order to develop beneficial health promotion programs, health education specialists need to understand the complexity of culture and be prepared to be culturally competent professionals [19]. They must have a level of understanding to the extent they are aware of the knowledge, attitudes, skills, and behaviors necessary for interacting effectively with ethnic/racially diverse populations. As the literature review suggests, academic preparation programs play an intricate role in developing the necessary skill sets for entry level practitioners. By having graduates enter the field deficient of the competencies needed to work in diverse communities, racial and ethnic minority populations may be left vulnerable as it relates to programming and poor health outcomes [15].

\section{Future Direction in Health Education}

Health Education academic program faculty and administrators must stay abreast of cultural competencies evaluated for practitioner certification. This not only ensures students entering the field obtain passing scores from these professional agencies, but also have the knowledge to have effective interactions with minority populations. In order to acquire these outcomes, curriculum and assignments must be continuously evaluated and modified to reflect current trends in the discipline. Academicians and practitioners have to be on the same accord in efforts to diminish and subsequently eliminate health disparities.

\section{Reference}

1. Office of Minority Health \& Health Disparities. About minority health. 2010. Available from: http://www.cdc.gov/omhd/AMH/AMH.htm.

2. Harvard Catalyst. Cultural competence in research. 2010. Available from: http://www.mfdp.med.harvard.edu/catalyst/p ublications/Cultural_Competence_Annotate d_Bibliography.pdf.

3. The Coalition of National Health Education Organizations. Code of ethics for the health education profession. 2011. Available from: http://www.cnheo.org/PDF\%20files/CODE \%20OF\%20ETHICS\%202011\%20Full.pdf.

4. Luquis R, Perez M, Young, K. Cultural competence development in health education professional preparation programs. Am J Health Behav. 2006;37(4):233-41.

5. Rojas-Guyler L, Wagner D, Chockalingam S. Latino cultural competence among Health Educators: Professional preparation implications. Am J Health Stud. 2006;21(2):99-106.

6. Tremethick M, Smit E. Preparing culturally competent health educators: The development and evaluation of a cultural immersion service-learning program. Electron J Health Educ. 2009;12:185-93.

7. Poirier, T, Butler L, Devraj R, Gupchup G, Santanello C, Lynch C. A cultural competency course for pharmacy students. Am J Pharm Educ. 2009;73(5):81.

8. Crandall S, George G, Marion G, Davis S. Applying theory to the design of cultural competency training for medical students: A case study. Acad Med. 2003;78(6):588-94.

9. Haack S, Phillips C. Teaching cultural competency through a pharmacy skills and applications course series. Am J Pharm Educ. 2012;76(2):27.

10. Alegria M. Training for research in mental health and HIV/AIDS among racial and ethnic minority populations: Meeting the needs of new investigators. Am J Public Health. 2009:99;26-30. 
11. Alvarez R, Vasquez E, Mayorga C, Feaster $\mathrm{D}$, Mitrani V. Increasing minority research participation through community organization outreach. West J Nurs Res. 2006;28:541-63.

12. Rabionet S, Santiago E, Zorilla C. A multifaceted mentoring model for minority researchers to address HIV health disparities. Am J Public Health. 2009;99(S1):65-70.

13. Gil E, Bob S. Culturally competent research: An ethical perspective. Clin Psychol Rev. 1999;19(1):45-55.

14. Bushy A. Conducting culturally competent rural nursing research. Annu Rev Nurs Res. 2008;26(1):221-36.

15. Betancourt J, Green A, Carrillo J, Park E. Cultural competence and health care disparities: Key perspectives and trends. Health Aff. 2005;24(2):499-505.

16. Echeverri M, Brookover C, Kennedy K. Nine constructs of cultural competence for curriculum development. Am J Pharm Educ. 2010;74(10):181.

17. Crenshaw K, Shewchuk R, Qu H, Staton L, Bigby J, Houston T, Allison J, Estrada C. What should we include in cultural competence curriculum? An emerging formative evaluation process to foster curriculum development. Acad Med. 2011;86(3):333-41.

18. Cuellar N, Brennan A, Vito K, de Leon Siantz, M. Cultural competence in the undergraduate nursing curriculum. J Prof Nurs. 2008;24(3):143-49.

19. American Association for Health Educators. Importance of cultural competence for health educators. 2006. Available from: http://aahperd.confex.com/aahperd/2006/fina lprogram/session_28773.htm. 